\title{
A GENERALIZED UPPER AND LOWER SOLUTION METHOD FOR SINGULAR DISCRETE BOUNDARY VALUE PROBLEMS FOR THE ONE-DIMENSIONAL $p$-LAPLACIAN
}

\author{
D. Q. JIANG, D. O'REGAN and R. P. AGARWAL \\ Received February 10, 2003 and, in revised form, June 26, 2003
}

\begin{abstract}
This paper presents new existence results for singular discrete boundary value problems for the one-dimension $p$-Laplacian. In particular our nonlinearity may be singular in its dependent variable and is allowed to change sign. Our results are new even for $p=2$.
\end{abstract}

\section{Introduction}

An upper and lower solution theory is presented for the singular discrete boundary value problem

$$
\left\{\begin{array}{l}
\Delta(\phi(\Delta y(i-1)))+q(i) f(i, y(i))=0, \quad i \in N=\{1, \ldots, T\} \\
y(0)=y(T+1)=0,
\end{array}\right.
$$

where $\phi(s)=|s|^{p-2} s, p>1, T \in\{1,2, \ldots\}, N^{+}=\{0,1, \ldots, T+1\}$ and $y: N^{+} \rightarrow \mathbb{R}$. Throughout this paper we will assume $f: N \times(0, \infty) \rightarrow \mathbb{R}$ is

2000 Mathematics Subject Classification. 34B16, 39A10.

Key words and phrases. Upper and lower solutions, discrete boundary value problem, existence, singular.

The work was supported by NNSF of China.

ISSN 1425-6908 C Heldermann Verlag. 
continuous. As a result our nonlinearity $f(i, u)$ may be singular at $u=0$ and may change sign.

Remark 1.1. Recall that a map $f: N \times(0, \infty) \rightarrow \mathbb{R}$ is continuous if it is continuous as a map of the topological space $N \times(0, \infty)$ into the topological space $\mathbb{R}$. Throughout this paper the topology on $N$ will be the discrete topology.

We will let $C\left(N^{+}, \mathbb{R}\right)$ denote the class of maps $u$ continuous on $N^{+}$(discrete topology), with norm $\|u\|=\max _{i \in N^{+}}|u(i)|$. By a solution to (1.1) we mean a $y \in C\left(N^{+}, \mathbb{R}\right)$ such that $y$ satisfies (1.1) for $i \in N$ and $y$ satisfies the boundary condition.

The literature on the one-dimensional $p$-Laplacian (when the nonlinearity is not singular in its dependent variable) is vast; see [18] and the references therein. Also the existence of solutions to singular boundary value problems in the continuous case have been studied in great detail in the literature (see $[6,7,8,10,13]$ (when $p=2$ ) and $[14,15]$ and the references therein). However, for the discrete case only a few papers have discussed boundary value problems. For example see $[4,5,11,12]($ when $p=2)$ and $[16,17]$. In $[16]$ the nonlinearity $f(i, u)$ may be singular at $u=0$ and may change sign, and the approach there is based on an argument initiated by Habets and Zanolin in [10]. In this paper a new approach is given which yields a very general existence theory for (1.1). Our results are new even for $p=2$. Not suprizingly our results improve considerable the results in [2] (when $p=2)$ and $[16,17]$.

\section{Some preliminary results}

In this section we present some results from literature which will be needed in Section 3.

We first state one well known result in [1].

Lemma 2.1 ([1]). Let $u \in C\left(N^{+}, \mathbb{R}\right)$ satisfy $u(i) \geq 0$ for $i \in N^{+}$. If $y \in C\left(N^{+}, \mathbb{R}\right)$ satisfies

$$
\left\{\begin{array}{l}
\Delta^{2} y(i-1)+u(i)=0, \quad i \in N=\{1,2, \ldots, T\} \\
y(0)=y(T+1)=0,
\end{array}\right.
$$

then

$$
y(i) \geq \mu(i)\|y\| \text { for } i \in N^{+}
$$

here

$$
\mu(i)=\min \left\{\frac{T+1-i}{T+1}, \frac{i}{T}\right\}
$$


Lemma 2.2. Let $[a, b]=\{a, a+1, \ldots, b\} \subset N$. If $y \in C\left(N^{+}, \mathbb{R}\right)$ satisfies

$$
\left\{\begin{array}{l}
\Delta^{2} y(i-1) \leq 0, \\
y(a-1) \geq 0, y(b+1) \geq 0,
\end{array} \quad i \in[a, b]\right.
$$

then $y(i) \geq 0$ for $i \in[a-1, b+1]=\{a-1, a, \ldots, b+1\} \subset N^{+}$.

Remark 2.1. Of course if $b=a$ then Lemma 2.2 is immediate.

Proof. Set

$$
Q(i)=y(a-1)+\frac{y(b+1)-y(a-1)}{b-a+2}(i+1-a), \quad i \in[a-1, b+1] .
$$

Let $y(i)=u(i)+Q(i)$. Then $\Delta^{2} u(i-1) \leq 0, i \in[a, b]$ and $u(a-1)=$ $u(b+1)=0$. Thus by Lemma $2.1, u(i) \geq 0$ for $i \in[a-1, b+1]$. Since $Q(i) \geq 0$, then $y(i) \geq 0$ for $i \in[a-1, b+1]$.

Lemma 2.3. Let $[a, b]=\{a, a+1, \ldots, b\} \subset N$. If $y \in C\left(N^{+}, \mathbb{R}\right)$ satisfies

$$
\left\{\begin{array}{l}
\Delta(\phi(\Delta y(i-1))) \leq 0, \\
y(a-1) \geq 0, \quad y(b+1) \geq 0,
\end{array} \quad i \in[a, b]\right.
$$

then $y(i) \geq 0$ for $i \in[a-1, b+1]=\{a-1, a, \ldots, b+1\} \subset N^{+}$.

Proof. Notice $\Delta(\phi(\Delta y(i-1))) \leq 0$ implies $\Delta^{2} y(i-1) \leq 0$ for $i \in[a, b]$, so the result follows from Lemma 2.2.

Lemma 2.4. Let $[a, b]=\{a, a+1, \ldots, b\} \subset N$. If $u, v \in C\left(N^{+}, \mathbb{R}\right)$ satisfy

$$
\left\{\begin{array}{l}
\Delta(\phi(\Delta u(i-1))) \leq \Delta(\phi(\Delta v(i-1))), \quad i \in[a, b] \\
u(a-1) \geq v(a-1), \quad u(b+1) \geq v(b+1),
\end{array}\right.
$$

then $u(i) \geq v(i)$ for $i \in[a-1, b+1]=\{a-1, a, \ldots, b+1\} \subset N^{+}$.

Proof. Suppose $u(i)<v(i)$ for some $i \in[a-1, b+1]$. Since $u(a-1) \geq$ $v(a-1), u(b+1) \geq v(b+1)$, the function $w(i)=u(i)-v(i)$ would have a negative minimum at a point $i_{0} \in[a, b]$. Hence $\Delta w\left(i_{0}-1\right) \leq 0$, i.e., $\Delta u\left(i_{0}-1\right) \leq \Delta v\left(i_{0}-1\right)$. Notice that

$$
\Delta(\phi(\Delta u(i-1))) \leq \Delta(\phi(\Delta v(i-1))), \quad i \in[a, b] .
$$

Sum both sides of the above inequality from $i_{0}$ to $i \in\left[i_{0}, b\right]=\left\{i_{0}, \ldots, b\right\}$ to get

$\phi(\Delta u(i))-\phi\left(\Delta u\left(i_{0}-1\right)\right) \leq \phi(\Delta v(i))-\phi\left(\Delta v\left(i_{0}-1\right)\right), \quad$ for all $i \in\left[i_{0}, b\right]$, 
and so we have

$\phi(\Delta u(i))-\phi(\Delta v(i)) \leq \phi\left(\Delta u\left(i_{0}-1\right)\right)-\phi\left(\Delta v\left(i_{0}-1\right)\right), \quad$ for all $i \in\left[i_{0}, b\right]$.

As a result

$$
\Delta w(i)=\Delta u(i)-\Delta v(i) \leq 0, \quad \text { for all } i \in\left[i_{0}, b\right],
$$

and so $w\left(i_{0}\right) \geq w(b+1) \geq 0$, a contradiction.

Consider the discrete boundary value problem

$$
\left\{\begin{array}{l}
\Delta(\phi(\Delta y(i-1)))+F(i, y(i))=0, \quad i \in N=\{1, \ldots, T\} \\
y(0)=A, y(T+1)=B,
\end{array}\right.
$$

where $A$ and $B$ are given real numbers, $\phi(s)=|s|^{p-2} s, p>1$. The following existence principle for problem (2.1) was established in $[16,17]$.

Lemma 2.5. Suppose that $F(i, u): N \times \mathbb{R} \rightarrow \mathbb{R}$ is continuous, and there exists $h \in C(N,[0, \infty))$ with $|F(i, u)| \leq h(i)$ for $i \in N$. Then (2.1) has a solution $y \in C\left(N^{+}, \mathbb{R}\right)$.

\section{Existence theory}

In this section we combine the ideas in [9] (when $p=2$ ) and [16] to obtain new results for the singular discrete boundary value problem

$$
\left\{\begin{array}{l}
\Delta(\phi(\Delta y(i-1)))+q(i) f(i, y(i))=0, \quad i \in N=\{1, \ldots, T\} \\
y(0)=y(T+1)=0,
\end{array}\right.
$$

where our nonlinearity $f$ may change sign. Our main result can be stated immediately.

Theorem 3.1. Let $n_{0} \in\{1,2, \ldots\}$ be fixed and suppose the following conditions are satisfied:

$$
\begin{gathered}
f: N \times(0, \infty) \rightarrow \mathbb{R} \text { is continuous } \\
\qquad q \in C(N,(0, \infty)) \\
\left\{\begin{array}{l}
\text { there exists a function } \alpha \in C\left(N^{+}, \mathbb{R}\right) \\
\text { with } \alpha(0)=\alpha(T+1)=0, \alpha>0 \text { on } N \text { such } \\
\text { that } q(i) f(i, \alpha(i)) \geq-\Delta(\phi(\Delta \alpha(i-1))) \text { for } i \in N
\end{array}\right.
\end{gathered}
$$

and

$$
\left\{\begin{array}{l}
\text { there exists a function } \beta \in C\left(N^{+}, \mathbb{R}\right) \text { with } \\
\beta(i) \geq \alpha(i) \text { and } \beta(i) \geq 1 / n_{0} \text { for } i \in N^{+} \text {with } \\
q(i) f(i, \beta(i)) \leq-\Delta(\phi(\Delta \beta(i-1))) \text { for } i \in N
\end{array}\right.
$$


Then (3.1) has a solution $y \in C\left(N^{+}, \mathbb{R}\right)$ with $y(i) \geq \alpha(i)$ for $i \in N^{+}$.

Proof. We begin with the discrete boundary value problem

$$
\left\{\begin{array}{l}
-\Delta(\phi(\Delta y(i-1)))=q(i) f_{n_{0}}^{\star}(i, y(i)), \quad i \in N \\
y(0)=y(T+1)=\frac{1}{n_{0}}
\end{array}\right.
$$

here

$$
f_{n_{0}}^{\star}(i, y)= \begin{cases}f(i, \alpha(i)), & y \leq \alpha(i) \\ f(i, y), & \alpha(i) \leq y \leq \beta(i) \\ f(i, \beta(i)), & y \geq \beta(i) .\end{cases}
$$

From Lemma 2.5 we know that $(3.6)$ has a solution $y_{n_{0}} \in C\left(N^{+}, \mathbb{R}\right)$. We first show

$$
y_{n_{0}}(i) \geq \alpha(i), \quad i \in N^{+} .
$$

Suppose $(3.7)$ is not true. Since $y_{n_{0}}(0)>\alpha(0)=0, y_{n_{0}}(T+1)>\alpha(T+1)=$ 0 , then there exists $[a, b]=\{a, a+1, \ldots, b\} \subset N$ such that

$$
y_{n_{0}}(i)<\alpha(i) \text { on }[a, b], y_{n_{0}}(a-1) \geq \alpha(a-1), y_{n_{0}}(b+1) \geq \alpha(b+1) .
$$

Thus for $i \in[a, b]$, we have

$$
\begin{aligned}
-\Delta\left(\phi\left(\Delta y_{n_{0}}(i-1)\right)\right) & =q(i) f_{n_{0}}^{\star}\left(i, y_{n_{0}}(i)\right)=q(i) f(i, \alpha(i)) \\
& \geq-\Delta(\phi(\Delta(\alpha(i-1))) .
\end{aligned}
$$

Since $y_{n_{0}}(a-1) \geq \alpha(a-1), y_{n_{0}}(b+1) \geq \alpha(b+1)$, it follows from Lemma 2.4 that $y_{n_{0}}(i) \geq \alpha(i)$ for $i \in[a-1, b+1]=\{a-1, a, \ldots, b+1\} \subset N^{+}$, a contradiction.

A similar argument shows

$$
y_{n_{0}}(i) \leq \beta(i) \quad \text { for } i \in N^{+} .
$$

Thus

$$
\alpha(i) \leq y_{n_{0}}(i) \leq \beta(i) \text { for } i \in N^{+} .
$$

Now proceed inductively to construct $y_{n_{0}+1}, y_{n_{0}+2}, y_{n_{0}+3}, \ldots$ as follows. Suppose we have $y_{k}$ for some $k \in\left\{n_{0}, n_{0}+1, n_{0}+2, \ldots\right\}$ with $\alpha(i) \leq$ $y_{k}(i) \leq y_{k-1}(i)$ for $i \in N^{+}$(here $y_{n_{0}-1}=\beta$ ). Then consider the discrete boundary value problem

$$
\left\{\begin{array}{l}
-\Delta(\phi(\Delta y(i-1)))=q(i) f_{k+1}^{\star}(i, y(i)), \quad i \in N \\
y(0)=\frac{1}{k+1}
\end{array}\right.
$$

here

$$
f_{k+1}^{\star}(i, y)= \begin{cases}f(i, \alpha(i)), & y \leq \alpha(i) \\ f(i, y), & \alpha(i) \leq y \leq y_{k}(i) \\ f\left(i, y_{k}(i)\right), & y \geq y_{k}(i)\end{cases}
$$


Now Lemma 2.5 guarantees that $(3.10)$ has a solution $y_{k+1} \in C\left(N^{+}, \mathbb{R}\right)$, and essentially the same reasoning as above yields

$$
\alpha(i) \leq y_{k+1}(i) \leq y_{k}(i) \text { for } i \in N^{+} .
$$

Thus for each $n \in\left\{n_{0}, n_{0}+1, \ldots\right\}$ we have

$$
\alpha(i) \leq y_{n}(i) \leq y_{n-1}(i) \leq \ldots \leq y_{n_{0}}(i) \leq \beta(i) \text { for } i \in N^{+} .
$$

Bolzano's theorem guarantees the existence of a subsequence $Z_{n_{0}}$ of integers and a function $y$ with $y_{n}$ converging to $y$ on $N^{+}$as $n \rightarrow \infty$ through $Z_{n_{0}}$. Also $y(0)=y(T+1)=0$. Now $y_{n}, n \in Z_{n_{0}}$, satisfies $y_{n}(i) \geq \alpha(i)>0$ for $i \in N$. Fix $i \in N$, and we obtain

$$
\begin{aligned}
\Delta\left(\phi\left(\Delta y_{n}(i-1)\right)\right) & =\phi\left(\Delta y_{n}(i)\right)-\phi\left(\Delta y_{n}(i-1)\right) \\
& =\phi\left(y_{n}(i+1)-y_{n}(i)\right)-\phi\left(y_{n}(i)-y_{n}(i-1)\right) \\
& \rightarrow \Delta(\phi(\Delta y(i-1))), i \in N, n \in Z_{n_{0}}, n \rightarrow \infty
\end{aligned}
$$

and

$$
f\left(i, y_{n}(i)\right) \rightarrow f(i, y(i)), i \in N, n \in Z_{n_{0}}, n \rightarrow \infty .
$$

Thus $\Delta(\phi(\Delta y(i-1)))+q(i) f(i, y(i))=0$ for $i \in N, y(0)=y(T+1)=0$. As a result $y \in C\left(N^{+}, \mathbb{R}\right)$ is a solution to (3.1) and also we have $\alpha(i) \leq$ $y(i) \leq \beta(i), i \in N^{+}$.

Suppose (3.2)-(3.4) hold, and in addition assume the following conditions are satisfied:

$$
\left\{\begin{array}{l}
q(i) f(i, y) \geq-\Delta(\phi(\Delta \alpha(i-1))) \\
\text { for }(i, y) \in N \times\{y \in(0, \infty): y<\alpha(i)\}
\end{array}\right.
$$

and

$$
\begin{cases}\text { there exists a function } \beta \in C\left(N^{+}, \mathbb{R}\right) \text { with } & \\ \beta(i) \geq \frac{1}{n_{0}} & \text { for } i \in N^{+} \text {with } \\ q(i) f(i, \beta(i)) \leq-\Delta(\phi(\Delta \beta(i-1))) & \text { for } i \in N .\end{cases}
$$

Then the result in Theorem 3.1 is again true. This follows immediately from Theorem 3.1 once we show $\beta(i) \geq \alpha(i)$ for $i \in N^{+}$. Suppose it is false. Since $\beta(0)>\alpha(0)=0, \beta(T+1)>\alpha(T+1)=0$, then there exists $[a, b]=\{a, a+1, \ldots, b\} \subset N$ such that

$$
\beta(i)<\alpha(i) \text { on }[a, b], \beta(a-1) \geq \alpha(a-1), \beta(b+1) \geq \alpha(b+1) .
$$

Thus for $i \in[a, b]$, we have

$$
q(i) f(i, \beta(i)) \geq-\Delta(\phi(\Delta \alpha(i-1))),
$$

and therefore

$$
-\Delta(\phi(\Delta \beta(i-1))) \geq-\Delta(\phi(\Delta(\alpha(i-1))), \quad i \in[a, b] .
$$


Since $\beta(a-1) \geq \alpha(a-1), \beta(b+1) \geq \alpha(b+1)$, it follows from Lemma 2.4 that $\beta(i) \geq \alpha(i)$ for $i \in[a-1, b+1]=\{a-1, a, \ldots, b+1\} \subset N^{+}$, a contradiction. Thus we have

Corollary 3.1. Let $n_{0} \in\{1,2, \ldots\}$ be fixed and suppose (3.2)-(3.4), (3.13) and (3.14) hold. Then (3.1) has a solution $y \in C\left(N^{+}, \mathbb{R}\right)$ with $y(i) \geq \alpha(i)$ for $i \in N^{+}$.

Next we discuss how to construct the lower solution $\alpha$ in (3.4) and in (3.13). Suppose the following condition is satisfied:

$$
\left\{\begin{array}{l}
\text { let } n \in\left\{n_{0}, n_{0}+1, \ldots\right\} \text { and associated with each } n \\
\text { there exists a constant } k_{0}>0 \text { such that for } i \in N \\
\text { and } 0<y \leq \frac{1}{n} \text { we have } q(i) f(i, y) \geq k_{0} .
\end{array}\right.
$$

Let $\alpha(i)=k v(i), i \in N^{+}$, where $v \in C\left(N^{+},[0, \infty)\right)$ is the solution of

$$
\left\{\begin{array}{l}
\Delta(\phi(\Delta v(i-1)))+1=0, \quad i \in N=\{1, \ldots, T\} \\
v(0)=v(T+1)=0
\end{array}\right.
$$

here

$$
0<k<\min \left\{\left[k_{0}\right]^{1 /(p-1)}, \frac{1}{n_{0}\|v\|}\right\} .
$$

Since $\Delta(\phi(\Delta v(i-1)))<0$ implies $\Delta^{2} v(i-1)<0$ for $i \in N$, it follows from Lemma 2.1 that $v(i) \geq \mu(i)\|v\|$ for $i \in N^{+}$. Thus, $\alpha(i) \leq 1 / n_{0}$, $-\Delta(\phi(\Delta \alpha(i-1)))=k^{p-1} \leq k_{0}, \alpha(0)=\alpha(T+1)=0, \alpha>0$ for $i \in N$, so (3.4) and (3.13) hold, since

$$
q(i) f(i, y) \geq k_{0} \geq-\Delta(\phi(\Delta \alpha(i-1))), \quad \text { for } i \in N, 0<y<\alpha(i),
$$

and

$$
q(i) f(i, \alpha(i)) \geq k_{0} \geq-\Delta(\phi(\Delta \alpha(i-1))), \quad i \in N .
$$

We combine this with Corollary 3.2 to obtain our next result.

Theorem 3.2. Let $n_{0} \in\{1,2, \ldots\}$ be fixed and suppose (3.2), (3.3), (3.14), and (3.15) hold. Then (3.1) has a solution $y \in C\left(N^{+}, \mathbb{R}\right)$ with $y(i)>0$ for $i \in N$.

Looking at Theorem 3.3 we see that the main difficulty when discussing examples is the construction of the $\beta$ in (3.14). Our next result replaces (3.14) with a growth condition which is natural from an application viewpoint and easy to check in practice. We first present the result in its full generality. 
Theorem 3.3. Let $n_{0} \in\{1,2, \ldots\}$ be fixed and suppose (3.2)-(3.4) hold. Also assume the following condition is satisfied:

$$
\left\{\begin{array}{l}
|f(i, y)| \leq g(y)+h(y) \text { on } N \times(0, \infty) \text { with } \\
g>0 \text { continuous and nonincreasing on }(0, \infty) \\
\text { and } h \geq 0 \text { continuous on }[0, \infty) \\
\frac{h}{g} \text { nondecreasing on }(0, \infty) .
\end{array}\right.
$$

Also suppose there exists a constant $M>\sup _{i \in N^{+}} \alpha(i)$ with

$$
b_{0}<\frac{1}{\phi^{-1}\left(1+\frac{h(M)}{g(M)}\right)} \int_{0}^{M} \frac{d y}{\phi^{-1}(g(y))}
$$

holding; here

$$
b_{0}=\max _{i \in N}\left(\sum_{j=1}^{i} \phi^{-1}\left(\sum_{z=j}^{i} q(z)\right), \sum_{j=i}^{T} \phi^{-1}\left(\sum_{z=i}^{j} q(z)\right)\right) .
$$

Then (3.1) has a solution $y \in C\left(N^{+}, \mathbb{R}\right)$ with $y(i) \geq \alpha(i)$ for $i \in N^{+}$.

Proof. Choose $\varepsilon>0, \varepsilon<M$, with

$$
\frac{1}{\phi^{-1}\left(1+\frac{h(M)}{g(M)}\right)} \int_{\varepsilon}^{M} \frac{d y}{\phi^{-1}(g(y))}>b_{0} .
$$

Without loss of generality assume $1 / n_{0}<\varepsilon$. We consider the discrete boundary value problem

$$
\left\{\begin{array}{l}
\Delta(\phi(\Delta y(i-1)))+q(i) g(y(i))\left(1+\frac{h(M)}{g(M)}\right)=0, i \in N, \\
y(0)=y(T+1)=\frac{1}{n_{0}} .
\end{array}\right.
$$

First we consider the modified discrete boundary value problem

$$
\left\{\begin{array}{l}
\Delta(\phi(\Delta y(i-1)))+q(i) g^{*}(y(i))\left(1+\frac{h(M)}{g(M)}\right)=0, i \in N \\
y(0)=y(T+1)=\frac{1}{n_{0}}
\end{array}\right.
$$

here

$$
g^{\star}(y)= \begin{cases}g\left(\frac{1}{n_{0}}\right), & y \leq \frac{1}{n_{0}} \\ g(y), & y \geq \frac{1}{n_{0}}\end{cases}
$$


Now $\left|g^{\star}(y)\right|=g^{\star}(y) \leq g\left(1 / n_{0}\right)$ for $y \in \mathbb{R}$, so Lemma 2.5 guarantees that (3.21) has a solution $\beta \in C\left(N^{+}, \mathbb{R}\right)$. Let $u(i)=\beta(i)-1 / n_{0}$ for $i \in N^{+}$. Then $\Delta(\phi(\Delta u(i-1)))=\Delta(\phi(\Delta \beta(i-1))) \leq 0$ for $i \in N$, and $u(0)=u(T+1)=0$. Lemma 2.3 guarantees that $u(i) \geq 0$, and so $\beta(i) \geq 1 / n_{0}$ for $i \in N^{+}$. Then $\beta$ is a solution to problem (3.20) also.

Now we claim that $\alpha(i) \leq \beta(i) \leq M, i \in N^{+}$. First we show

$$
\beta(i) \geq \alpha(i), \quad i \in N^{+} .
$$

Suppose (3.22) is false. Since $\beta(0)=\beta(T+1)=1 / n_{0}>\alpha(0)=\alpha(1)=0$, then there exists $[a, b]=\{a, a+1, \ldots, b\} \subset N$ such that

$$
\beta(i)<\alpha(i) \text { on }[a, b], \quad \beta(a-1) \geq \alpha(a-1), \quad \beta(b+1) \geq \alpha(b+1) .
$$

Thus for $i \in[a, b]$, we have from (3.20) and $M>\sup _{i \in N^{+}} \alpha(i)$ that

$$
\begin{aligned}
-\Delta(\phi(\Delta \beta(i-1))) & =q(i) g(\beta(i))\left(1+\frac{h(M)}{g(M)}\right) \\
& \geq q(i) g(\alpha(i))\left(1+\frac{h(\alpha(i))}{g(\alpha(i))}\right) \\
& \geq q(i) f(i, \alpha(i)) \geq-\Delta(\phi(\Delta \alpha(i-1))) .
\end{aligned}
$$

Since $\beta(a-1) \geq \alpha(a-1), \beta(b+1) \geq \alpha(b+1)$, it follows from Lemma 2.4 that $\beta(i) \geq \alpha(i)$ for $i \in[a-1, b+1]=\{a-1, a, \ldots, b+1\} \subset N^{+}$, a contradiction.

Next we show

$$
\beta(i) \leq M, \quad i \in N^{+} .
$$

Since $\Delta(\phi(\Delta \beta(i-1))) \leq 0$ on $N$ implies $\Delta^{2} \beta(i-1) \leq 0$ on $N$, then $\beta(i) \geq 1 / n_{0}$ on $N^{+}$and there exists $i_{0} \in N$ with $\Delta \beta(i) \geq 0$ on $\left[0, i_{0}\right)=$ $\left\{0,1, \ldots, i_{0}-1\right\}$ and $\Delta \beta(i) \leq 0$ on $\left[i_{0}, T+1\right)=\left\{i_{0}, i_{0}+1, \ldots, T\right\}$, and $\beta\left(i_{0}\right)=\|\beta\|$.

Also notice that for $z \in N$, we have

$$
-\Delta(\phi(\Delta \beta(z-1)))=g(\beta(z))\left(1+\frac{h(M)}{g(M)}\right) q(z) .
$$

We sum the equation (3.24) from $j+1\left(0 \leq j<i_{0}\right)$ to $i_{0}$ to obtain

$$
\phi(\Delta \beta(j))=\phi\left(\Delta \beta\left(i_{0}\right)\right)+\left(1+\frac{h(M)}{g(M)}\right) \sum_{z=j+1}^{i_{0}} g(\beta(z)) q(z) .
$$

Since $\Delta \beta\left(i_{0}\right) \leq 0$, and $\beta(z) \geq \beta(j+1)$ when $j+1 \leq z \leq i_{0}$, we have

$$
\phi[\Delta \beta(j)] \leq g(\beta(j+1))\left(1+\frac{h(M)}{g(M)}\right) \sum_{z=j+1}^{i_{0}} q(z), \quad j<i_{0},
$$


i.e.,

$$
\frac{\Delta \beta(j)}{\phi^{-1}(g(\beta(j+1)))} \leq \phi^{-1}\left(1+\frac{h(M)}{g(M)}\right) \phi^{-1}\left(\sum_{z=j+1}^{i_{0}} q(z)\right), j<i_{0} .
$$

Since $g(\beta(j+1)) \leq g(u) \leq g(\beta(j))$ for $\beta(j) \leq u \leq \beta(j+1)$ when $j<i_{0}$, we have

$$
\int_{\beta(j)}^{\beta(j+1)} \frac{d u}{\phi^{-1}(g(u))} \leq \frac{\Delta \beta(j)}{\phi^{-1}(g(\beta(j+1)))}, \quad j<i_{0} .
$$

It follows from (3.25) and (3.26) that

$$
\int_{\beta(j)}^{\beta(j+1)} \frac{d u}{\phi^{-1}(g(u))} \leq \phi^{-1}\left(1+\frac{h(M)}{g(M)}\right) \phi^{-1}\left(\sum_{z=j+1}^{i_{0}} q(z)\right), \quad j<i_{0},
$$

and then we sum the above from 0 to $i_{0}-1$ to obtain

$$
\begin{aligned}
\int_{1 / n_{0}}^{\beta\left(i_{0}\right)} \frac{d u}{\phi^{-1}(g(u))} & \leq \phi^{-1}\left(1+\frac{h(M)}{g(M)}\right) \sum_{j=0}^{i_{0}-1} \phi^{-1}\left(\sum_{z=j+1}^{i_{0}} q(z)\right) \\
& =\phi^{-1}\left(1+\frac{h(M)}{g(M)}\right) \sum_{j=1}^{i_{0}} \phi^{-1}\left(\sum_{z=j}^{i_{0}} q(z)\right) .
\end{aligned}
$$

Similarly, we sum the equation (3.24) from $i_{0}$ to $j\left(i_{0} \leq j<T+1\right)$ to obtain

$$
-\phi(\Delta \beta(j))=-\phi\left(\Delta \beta\left(i_{0}-1\right)\right)+\left(1+\frac{h(M)}{g(M)}\right) \sum_{z=i_{0}}^{j} g(\beta(z)) q(z), s \geq t_{0} .
$$

Since $\Delta \beta\left(i_{0}-1\right) \geq 0$, we have

$$
\frac{-\Delta \beta(j)}{\phi^{-1}(g(\beta(j)))} \leq \phi^{-1}\left(1+\frac{h(M)}{g(M)}\right) \phi^{-1}\left(\sum_{z=i_{0}}^{j} q(z)\right), \quad j \geq i_{0} .
$$

So we have

$\int_{\beta(j+1)}^{\beta(j)} \frac{d u}{\phi^{-1}(g(u))} \leq \frac{-\Delta \beta(j)}{\phi^{-1}(g(\beta(j)))} \leq \phi^{-1}\left(1+\frac{h(M)}{g(M)}\right) \phi^{-1}\left(\sum_{z=i_{0}}^{j} q(z)\right), j \geq i_{0}$,

and then we sum the above from $i_{0}$ to $T$ to obtain

$$
\int_{1 / n_{0}}^{\beta\left(i_{0}\right)} \frac{d u}{\phi^{-1}(g(u))} \leq \phi^{-1}\left(1+\frac{h(M)}{g(M)}\right) \sum_{j=i_{0}}^{T} \phi^{-1}\left(\sum_{z=i_{0}}^{j} q(z)\right) .
$$

Now (3.27) and (3.28) imply

$$
\int_{\varepsilon}^{\beta\left(i_{0}\right)} \frac{d u}{\phi^{-1}(g(u))} \leq \int_{1 / n_{0}}^{\beta\left(i_{0}\right)} \frac{d u}{\phi^{-1}(g(u))} \leq b_{0} \phi^{-1}\left(1+\frac{h(M)}{g(M)}\right) .
$$


This together with (3.19) implies $\|\beta\|=\beta\left(i_{0}\right) \leq M$.

Observe that

$$
\begin{aligned}
f(i, \beta(i)) & \leq g(\beta(i))\left(1+\frac{h(\beta(i))}{g(\beta(i))}\right) \\
& \leq g(\beta(i))\left(1+\frac{h(M)}{g(M)}\right), \quad i \in N .
\end{aligned}
$$

Thus we have $\beta(i) \geq 1 / n_{0}$ and $\beta(i) \geq \alpha(i)$ for $i \in N^{+}$with

$$
-\Delta\left(\phi(\Delta(\beta(i-1)))=q(i) g(\beta(i))\left(1+\frac{h(M)}{g(M)}\right) \geq q(i) f(i, \beta(i)), i \in N,\right.
$$

so that $\beta(i)$ satisfies (3.5). The result follows from Theorem 3.1.

Combining Theorem 3.4 with the comments before Theorem 3.3 yields the following theorem.

Theorem 3.4. Let $n_{0} \in\{1,2, \ldots\}$ be fixed and suppose (3.2), (3.3), (3.15) and (3.17) hold. In addition assume there is a constant $M>0$ with (3.18) holding. Then (3.1) has a solution $y \in C\left(N^{+}, \mathbb{R}\right)$ with $y(i)>0$ for $i \in N$.

Proof. This follows immediately from Theorem 3.4 once we show there exists $\alpha \in C\left(N^{+}, \mathbb{R}\right)$ such that (3.4) hold, and

$$
M>\alpha(i) \text { for each } i \in N^{+} \text {. }
$$

Let $\alpha(i)=k v(i), i \in N^{+}$, where $v$ is defined by (3.16), and

$$
0<k<\min \left\{\left[k_{0}\right]^{1 /(p-1)}, \frac{1}{n_{0}\|v\|}, \frac{M}{\|v\|}\right\} .
$$

Thus, $\alpha(i) \leq 1 / n_{0},-\Delta(\phi(\Delta \alpha(i-1)))=k^{p-1} \leq k_{0}, \alpha(0)=\alpha(T+1)=0$, $\alpha>0$ for $i \in N$ with (3.4) holding, since

$$
q(i) f(i, \alpha(i)) \geq k_{0} \geq-\Delta(\phi(\Delta \alpha(i-1))), \quad i \in N .
$$

Then $\alpha \in C\left(N^{+}, \mathbb{R}\right)$ and (3.4), and (3.29) hold.

Next we present an example which illustrates how easily the theory is applied in practice.

Example 3.1. The boundary value problem

$$
\left\{\begin{array}{l}
\Delta(\phi(\Delta y(i-1)))+\sigma\left([y(i)]^{-\alpha}+[y(i)]^{\beta}+\sin \frac{8 \pi i}{T}\right), \quad i \in N \\
y(0)=y(T+1)=0
\end{array}\right.
$$


with $\alpha>0, \beta \geq 0$ and $\sigma>0$ has a solution $y \in C\left(N^{+}, \mathbb{R}\right)$ with $y(i)>0$ for $i \in N$, if

$$
\sigma<\left[\frac{p-1}{b_{1}(\alpha+p-1)}\right]^{p-1} \sup _{c \in(0, \infty)} \frac{c^{\alpha+p-1}}{1+c^{\alpha}+c^{\alpha+\beta}}
$$

here

$$
b_{1}=\max _{i \in N}\left(\sum_{j=1}^{i}(i-j+1)^{1 /(p-1)}, \sum_{j=i}^{T}(j-i+1)^{1 /(p-1)}\right) .
$$

To see this we will apply Theorem 3.5 with

$$
q(i)=\sigma, \quad g(u)=u^{-\alpha}, \quad h(u)=u^{\beta}+1 .
$$

Clearly (3.2), (3.3), (3.15) and (3.17) hold. Also notice that (3.31) implies that there exists $M>0$ such that

$$
\sigma<\left[\frac{p-1}{b_{1}(\alpha+p-1)}\right]^{p-1} \frac{M^{\alpha+p-1}}{1+M^{\alpha}+M^{\alpha+\beta}} ;
$$

and so (3.18) holds.

Thus all the conditions of Theorem 3.5 are satisfied so existence is guaranteed.

Remark 3.1. If $\beta<p-1$ then (3.31) is automatically satisfied.

\section{References}

[1] Agarwal, R. P., O'Regan, D., Nonpositive discrete boundary value problems, Nonlinear Anal. 39 (2000), 207-215.

[2] Agarwal, R. P., O'Regan, D., Singular discrete boundary value problems, Appl. Math. Lett. 12 (1999), 127-131.

[3] Agarwal, R. P., O'Regan, D., Boundary value problems for discrete equations, Appl. Math. Lett. 10 (1997), 83-89.

[4] Agarwal, R. P., O'Regan, D., Singular discrete $(n, p)$ boundary value problems, Appl. Math. Lett. 12 (1999), 113-119.

[5] Agarwal, R. P., O'Regan, D., Wong, P. J. Y., Positive Solutions of Differential, Difference and Integral Equations, Kluwer Acad. Publ., Dordrecht, 1999.

[6] Agarwal, R. P., O'Regan, D., Singular initial and boundary value problems with sign changing nonlinearities, IMA J. Appl. Math. 65 (2000), 173-198.

[7] Agarwal, R. P., O'Regan, D., Some new existence results for singular problems with sign changing nonlinearities, J. Comput. Appl. Math. 113 (2000), 1-15.

[8] Agarwal, R. P., O'Regan, D., Lakshmikantham, V., Leela, S., Existence of positive solutions for singular initial and boundary value problems via the classical upper and lower solution approach, Nonlinear Anal. 50 (2002), 215-222.

[9] Agarwal, R. P., O'Regan, D., Lakshmikantham, V., Leela, S., An upper and lower solution theory for singular Emden-Fowler equations, Nonlinear Anal.: Real World Appl. 3 (2002), 275-291.

[10] Habets, P., Zanolin, F., Upper and lower solutions for a generalized Emden-Fowler equation, J. Math. Anal. Appl. 181 (1994), 684-700. 
[11] Henderson, J., Singular boundary value problems for difference equations, Dynam. Systems Appl. 1 (1992), 271-282.

[12] Henderson, J., Singular boundary value problems for higher order difference equations, World Congress on Nonlinear Analysts 1992 (Tampa, FL, August 1992), Vol I-IV, 1139-1150, de Gruyter, Berlin, 1996.

[13] Jiang, D. Q., Upper and lower solutions for a superlinear singular boundary value problem, Comput. Math. Appl. 41 (2001), 563-569.

[14] Jiang, D. Q., Upper and lower solutions method and a superlinear singular boundary value problem for the one-dimension p-Laplacian, Comput. Math. Appl. 42 (2001), 927-940.

[15] Jiang, D. Q., Gao, W., Upper and lower solution method and a singular boundary value problem for the one-dimension p-Laplacian, J. Math. Anal. Appl. 252 (2000), 631-648.

[16] Jiang, D. Q., Pang, P. Y. H., Agarwal, R. P., Upper and lower solutions method and a superlinear singular discrete boundary value problem, Dynam. Systems Appl. (to appear).

[17] Jiang, D. Q., Zhang, L., O'Regan, D., Agarwal, R. P., Existence theory for single and multiple solutions to singular positone discrete Dirichlet boundary value problems to the one-dimensional p-Laplacian, Arch. Math. 40 (2004), 367-381.

[18] Manasevich, R., Zanolin, F., Time mappings and multiplicity of solutions for the one-dimensional p-Laplacian, Nonlinear Anal. 21 (1993), 269-291.

DAQING JIANG

DEPARTMENT OF MATHEMATICS

Northeast Normal University

ChangChun 130024

P. R. CHINA
DONAL O'REGAN

Department of Mathematics NATIONAL UNIVERSITY OF IRELAND

GALWAY

IRELAND

E-MAIL: DONAL.OREGAN@UNIGALWAY.IE

Ravi P. Agarwal

Department of Mathematical Science

Florida Institute of TeChNology

Melbourne, FL 32901-6975

USA

E-MAIL: AGARWAL@FIT.EDU 